\title{
PTX3 activates classic complement pathway and mediates phagocytosis of cholesterol-induced apoptotic macrophages in atherosclerosis
}

tang-meng guo

institution of geriatrics,union hospital,tongji medical college https://orcid.org/0000-0002-0463-8349

li ke

institution of geriatrics,union hospital,tongji medical college

bei cheng ( $\nabla$ chengbei2014@126.com )

Research

Keywords: PTX3, C1q, classic complement pathway, efferocytosis, atherosclerosis

Posted Date: February 13th, 2020

DOI: https://doi.org/10.21203/rs.2.23439/v1

License: (c) (i) This work is licensed under a Creative Commons Attribution 4.0 International License.

Read Full License 


\section{Abstract}

Backgrounds/Aims: Apoptotic macrophages are removed by neighboring phagocytes (efferocytosis), which is an important event in advanced atherosclerosis. We reported the long pentraxin 3 (PTX3) located at the membrane of late apoptotic macrophages, mediates efferocytosis in a cell model of advanced atherosclerosis. However, the mechanism underlying PTX3-mediated apoptotic cell clearance in atherogenesis is unclear.

Methods: We modeled macrophage apoptosis in advanced plaques by incubating macrophages (peritoneal macrophages isolated from C57 mice) with free cholesterol (free cholesterol-induced apoptotic macrophages, FC-AMs). FC-AMs were added to a monolayer of fresh phagocytes $\llbracket$ macrophages $₫$ to study the engulfment response. The percentage of phagocytes that ingested FC-AMs was quantified by using confocal microscopy. C1q and C3b were detected by using fluorescenceactivated cell sorter (FACS) and the confocal microscopy.

Results: We found that PTX3 significantly enhances complement C1q binding on FC-AMs and was correlated with improved activating of classic complement pathway. C3b was the characteristic component of activating of classic complement pathway, displayed the increased deposition on FC-AMs and mediated the phagocytosis of FC-AMs. This study replicated the role of PTX3-mediated efferocytosis, explored the mechanism of PTX3 involving efferocytosis in detail and provided theoretical evidences for PTX3-mediated cardio-protective functions in atherosclerosis.

Conclusion: PTX3 activated classic complement pathway and mediated phagocytosis of cholesterolinduced apoptotic macrophages in atherosclerosis.

\section{Background}

Immune responses participate in several phases of atherosclerosis[1]. Both adaptive immunity and innate immunity are believed to tightly regulate atherogenesis. PTX3, an essential component of the humoral arm of innate immunity, is a super-family of acute-phase proteins highly conserved during evolution and involves the development of atherosclerosis[2]. PTX3 is rapidly produced and released by several cell types, particularly mononuclear phagocytes, dendritic cells, fibroblasts, osteoblasts and endothelial cells[3,4]. PTX3 is produced obviously by macrophages and endothelial cells in advanced atherosclerotic lesions[2,5]. Mice lacking PTX3 showed more pronounced inflammatory profile in the vascular wall, an increased macrophage accumulation within the plaque, a greater no-reflow area in a model of acute myocardial infarction, however, all of which could be reversed by exogenous PTX3[6,7]. The above data support the cardio-protective function of PTX3.

However, the mechanism underlying PTX3-mediated cardio-protective function is also unclear. Our previous studies modeled macrophage apoptosis in advanced plaques by incubating macrophages (peritoneal macrophages isolated from C57 mice) with free cholesterol (free cholesterol-induced apoptotic macrophages, FC-AMs) [8]. Our results showed PTX3 was located at the membrane of late 
apoptotic macrophages and mediates the phagocytosis of apoptotic macrophages(efferocytosis)[8]. However, the components interacting with PTX3 and mediating phagocytosis are also unknown till now.

Complement plays a key role in atherosclerosis[9]. Cholesterol crystals can activate the complement system and induce an inflammatory response in the development of atherosclerosis[10]. In atherosclerotic lesions, expression of C1q occurs during differentiation of monocytes to DCs and macrophages and found be important in playing a protective role in early lesion formation in LDL receptor deficient mice[11]. C1q promotes macrophage survival during ingestion of excess cholesterol, as well as improves foam cell efferocytic function[12]. Surface-immobilized monomer C-reactive protein was able to initiate classical pathway activation and lead to C3 turnover[13]. A cell surface receptor for C3b is also expressed by macrophages in human atherosclerosis, which is involved in the clearance of circulating opsonized particles by hepatic Kupffer cells from the circulation[14,15]. If PTX3 interacts with complement and mediates phagocytosis are also unknown till now.

In this study, we would explore the mechanism of PTX3 activates C1q-mediated classic complement pathway and mediates phagocytosis of cholesterol-induced apoptotic macrophages, which may be important in providing insight into the protective role of PTX3 in atherosclerosis.

\section{Materials And Methods}

\section{Reagents}

PTX3 was purchased from Sigma-Aldrich (Cat. No. SRP6323, St. Louis, Missouri, USA). Anti-PTX3 monoclonal antibody (mAb) was purchased from Lifespan Bioscience Inc. (Cat. No. LS-C198038, Seattle,WA, USA), Abcam Biotech. (Cat. No. ab125007, Cambridge, Massachusetts, USA), and Santa Cruz (Cat. No. sc-373951, Biothch., Santa Cruz, CA, USA). ACAT inhibitor, S-58035, was purchased from SigmaAldrich (Cat. No. S9318, St. Louis, Missouri, USA). Acetyl-LDL was purchased from Yeasen biotech Co., Ltd. (Shanghai, China). Roswell park memorial institute 1640 (RPMI-1640) was purchased from Gibco BRL. Co. Ltd. (Grand Island,New York, USA). C1q protein was purchased from Fitzgerald Industries International (Cat. No. 32-AC02, Bremerton, WA, USA). Anti-C1q mAb was purchased from Santa Cruz (Cat. No. sc-58920 PE, Santa Cruz, CA, USA) and Abcam Biotech. (Cat. No. ab182451, Cambridge, Massachusetts, USA). C1q-Deficient Normal Human Serum (C1qD NHS) was purchased from Calbiochem (Cat. No. 234401, Merck, KGaA, Darmstadt, Germany). Anti-C3b mAb was purchased from Bioss (Cat. No. bs-4871r, Shanghai, China). Alexa Fluor-labeled IgG was purchased from Abcam Biotech. (Cat. No. ab150079, Cambridge, Massachusetts, USA). Cy3-labeled IgG, the membrane probe DiL and nucleic acid staining agent 4',6-diamidino-2-phenylindole (DAPI) were purchased from Beyotime Biotech Co. Ltd. (Cat. No. A0521, Shanghai, China). Non-enzymatic cell dissociation solution was purchased from M\&C Gene Technology (Beijing, China).

\section{Cells}


Concanavalin A-elicited macrophages were obtained from Female C57BL6/J mice (8-10 weeks of age) that were intraperitoneally injected with $0.5 \mathrm{ml}$ of phosphate-buffered saline (PBS) containing $40 \mu \mathrm{g}$ of concanavalin A[16]. All animal care and experimental procedures were approved by the ethical committee of Tongji Medical College, Huazhong University of Science and Technology, and followed the institutional guideline and ethical standard. Macrophages were harvested 3 days later by the peritoneal lavage. The macrophages were cultured in RPMI 1640 supplementing with $10 \%$ fetal bovine serum for $24-48 \mathrm{~h}$ until reaching the confluence. Live macrophages were obtained from macrophages that were cultured for 24$48 \mathrm{~h}$. FC-AMs were generated by incubating the macrophages with $100 \mu \mathrm{g} / \mathrm{ml}$ of acetyl-LDL and $10 \mu \mathrm{g} / \mathrm{ml}$ of the compound 58035 for $16-20 \mathrm{~h}$.

\section{Fluorescence-activated Cell Sorter (FACS) Analysis Detection of C1q}

The binding of C1q to FC-AMs was analyzed in the presence of C1q, PTX3 or anti-PTX3 mAbs. To harvest these FC-AMs, a nonenzymatic cell dissociation solution was used to treat FC-AMs at $37^{\circ} \mathrm{C}$ for $5-10 \mathrm{~min}$. Next the FC-AMs were incubated independently with C1q, PTX3 or anti-PTX3 mAbs for 30 min at $4{ }^{\circ} \mathrm{C}$. Then the FC-AMs were washing with RPMI 1640 supplementing with $10 \%$ fetal bovine serum. After incubating with goat serum, the FC-AMs were incubated with anti-C1q mAbs for $30 \mathrm{~min}$ at $4^{\circ} \mathrm{C}$. After washing, the FC-AMs were incubated with Alexa Fluor-labeled IgG or Cy3-labeled IgG at $4^{\circ} \mathrm{C}$ for $30 \mathrm{~min}$. C1q on FC-AMs was analyzed using FACS.

\section{Confocal Microscopy Analysis Detection of $\mathrm{C} 1 \mathrm{q}$ and $\mathrm{C} 3 \mathrm{~b}$}

Immunofluorescence assay was analyzed according to the manufacturer's instructions. FC-AMs were fixed with $4 \%$ paraformaldehyde for 15 min at room temperature. After incubation with goat serum, FCAMs were incubated with anti-C1q mAbs or anti-C3b mAbs for $12 \mathrm{~h}$ at $4^{\circ} \mathrm{C}$. After washing, FC-AMs were incubated with Alexa Fluor-labeled or Cy3-labeled IgG at room temperature for $60 \mathrm{~min}$. FC-AMs were washed with PBS for three times. Confocal microscopy was used to analyze the fluorescence.

\section{Phagocytosis}

FC-AMs were labeled with the membrane probe DiL at room temperature for $15 \mathrm{~min}$. Then, they were added to a monolayer of fresh phagocytes (macrophages). After incubation with the phagocytes at $37^{\circ} \mathrm{C}$ for 30 min, non-ingested FC-AMs were removed by vigorous washing. The phagocytes were labeled with DAPI and fixed with $4 \%$ paraformaldehyde and viewed by using the confocal microscopy. The percentage of phagocytes that ingested FC-AMs was quantified by confocal microscopy (blind counting). 


\section{Statistical Analysis}

The data were leveled as the mean \pm standard deviation and the comparisons between the groups were performed using the independent sample't tests. The statistical significance was defined as $P<0.05$.

\section{Results}

\section{Binding of C1q to apoptotic cells}

The binding of C1q to apoptotic cells was analyzed in the absence or presence of C1q with FACS and Confocal Microscopy analysis. We used the anti-C1q-mAbs to bind the membrane surface protein C1q. These antibodies were recognized using goat anti-mouse-Cy3-labeled IgG (red-orange). The mean optical density representing $\mathrm{C} 1 \mathrm{q}$ levels were higher with pre-incubation with $\mathrm{C} 1 \mathrm{q}$ as shown in Fig. 1A-D. The percentages of C1q positive cells were increased with pre-incubation with C1q (Fig. 1E).

\section{PTX3 enhances C1q binding on apoptotic cells}

We observed the effects of PTX3 on C1q binding on apoptotic cells in the presence of PTX3 or anti-PTX3 mAbs by confocal microscopy and FACS. Mean optical density representing C1q levels in pre-incubation with PTX3 groups was $20.06 \pm 3.0$, higher than FC-AMs groups' (the mean optical density was $13.45 \pm$ 2.54) (Fig. 2A-D). This observation was in keeping with the result of the FACS. FC-AMs pre-incubated with PTX3 showed more percentages of C1q positive cells. These effects could be reversed by the preincubation with anti-PTX3 mAbs. Compared to the FC-AMs groups (the percentage of C1q positive cells was $0.81 \% \pm 0.04 \%$ ), the percentage of C1q positive cells (pre-incubation with PTX3 groups and antiPTX3 mAbs groups) was $0.90 \% \pm 0.29 \%$ and $0.75 \% \pm 0.44 \%$, respectively (mean $\pm S E, n=3, P<0.01$ ) (Fig. 2E). It suggested that PTX3 enhances C1q binding on apoptotic cells.

\section{C1q-mediated Activating Of Classic Complement Pathway}

We incubated FC-AMs alone or with NHS, C1q D NHS, anti-C1q mAbs, C1q and assessed the C3b deposition. Pre-incubation with C1q resulted in obvious C3b deposition on the FC-AMs. When the cells were incubated concomitantly with C1q D NHS and anti-C1q mAbs, an obvious decrease in C3b deposition was observed (Fig. 3).

\section{PTX3 Enhances C1q-mediated Activating Of Classic Complement Pathway}

We observed the complement activation by evaluating the C3b deposition in the presence of PTX 3 or antiPTX3 mAbs. These results were expressed by mean optical density (red-orange). Pre-incubation with 
PTX3 resulted in significant C3b deposition on the FC-AMs (Fig. 4B). The process was reversed by the anti-PTX3 mAbs, leading to the reduced C3b deposition (Fig. 4C). The statistical results of mean optical density presented as mean \pm SD were shown in Fig. $4 \mathrm{E}$.

\section{The activating of classic complement pathway mediates phagocytosis of FC-AMs}

We studied the role of the classical complement activation component C3b on the phagocytosis of FCAMs by employing the macrophage. Pre-incubation with anti-C3b mAbs resulted in phagocytosis decline of FC-AMs (Fig. 5A, B). In control groups, the percentage of phagocytosis was $94 \% \pm 1 \%$. Compared to the control groups, the percentage of phagocytosis in anti-C3b mAbs groups was lower significantly ( $83 \% \pm$ $2 \%)($ mean $\pm S E, n=2, P<0.05)$ (Fig. 5B).

\section{Discussion}

LDL is engulfed by the macrophages in vascular wall. Free cholesterol-induced apoptotic macrophages are formed and express the PTX3 on their membrane. Increased PTX3 recruits the binding of C1q (the initial component of complement). The next changing of $\mathrm{C} 1 \mathrm{q}$ structure activates the classic complement pathway, and the increased C3b is deposited on the membrane of apoptotic cells (FC-AMs). C3b is recognized by the complement receptor 1 (CR1) on phagocytes and mediates the process of the phagocytosis. The series processes are summarized in the Fig. 6.

Emerging evidence indicates the preserved roles of efferocytosis in atherosclerosis throughout the healthy human body $[17,18]$. It is not yet understood why efferocytosis is dysfunctional within the atherosclerotic plaque. Just as the previously study reported that there are three following aspects: (1) overwhelming apoptosis within the plaque, (2) impairment in the phagocytic capacity of lesional macrophages or (3) reduced edibility of apoptotic vascular cells[19].

Several bridging molecules involving in the reduced edibility of efferocytosis have now been experimentally linked to the growing of atherosclerotic plaque. These factors include complement C1q, growth arrest specific 6 (gas-6), milk fat globule-epidermal growth factor 8 (mfge8) and transglutaminase $2(\operatorname{tg} 2)[20]$. Complement $\mathrm{C} 1 \mathrm{q}$, the first component of the classical pathway, initiates the activation of the classic pathway[21]. Then, pyrolysis components, C3b and C4b, produced from the complement activation accumulate on the apoptotic cells, mediate the efferocytosis of apoptotic cells[22]. C1q gene-targeted mice confirmed that the classic complement pathway plays a role in apoptotic cell clearance in atherosclerosis[23]. Meanwhile, the defective apoptotic cell clearance increases the lesion development[23]. PTX3 and C1q belong to the humoral arm of the innate immune system. PTX3 enhances C1q deposition and triggers the subsequent complement activation on apoptotic cells[24]. These are consistent with our study that showed that PTX3 enhanced the C1q binding on FCAMs in atherosclerotic cell model. 
In atherosclerotic cell model, we also observed the C1q involved activation of C3. We found that the activated complement C3b was increased on FC-AMs when C1q was present. PTX3 enhanced the process of $\mathrm{C} 1 \mathrm{q}$ involved activation of C3. The PTX3 enhanced activation of classic complement pathway were also confirmed in the other previous studies[24,25]. It gave an evidence for the theory that PTX3 binding $\mathrm{C} 1 \mathrm{q}$ mediates activation of classic complement pathway in atherosclerosis. However it is not clear the effect of classic complement pathway activation on atherosclerosis. Then we observed the effect of classic complement pathway activation on efferocytosis of FC-AMs. The levels of pyrolysis components $\mathrm{C} 3 \mathrm{~b}$ and $\mathrm{C} 4 \mathrm{~b}$, the central hub of the complement system, represented the activation of classic complement pathway[23]. C3b/C4b on desialated sheep erythrocytes involved in their phagocytosis when binding on $\mathrm{C} 3 \mathrm{~b} / \mathrm{C} 4 \mathrm{~b}$ receptor (CR1) on macrophages. The process was inhibited by treating macrophages with a monoclonal antibody to CR1 $[7,26,27]$. We also observed that C3b played an important part in efferocytosis of FC-AMs, however, which was inhibited by a monoclonal antibody of C3b. Taken together, there is convincing evidence to suggest that PTX3 mediates phagocytosis of apoptotic macrophages by activating classic complement pathway in an atherosclerotic cell model.

\section{Conclusion}

Efferocytosis has tremendous potential as both a therapeutic and diagnostic target[28]. The processes of macrophage apoptosis and defective efferocytic clearance of these apoptotic cells may play a critical role in plaque progression of coronary artery disease (CAD)[20]. Our previous study confirmed that PTX3 is located at the membrane of late apoptotic macrophages and mediates phagocytosis of apoptotic macrophages[8], which is associated with activating classic complement pathway in an atherosclerotic cell model. Understanding of the indepth molecular and cellular biology of efferocytosis would be beneficial to the open up novel opportunities for mechanism-based therapy. Our another published study showed that PTX3 promoter methylation is associated with the PTX3 plasma levels in CAD[29]. Compared to neutrophil to lymphocyte ratio, matrix metalloprotein 9, and interleukin-6, PTX3 displayed greater area under the receiver operating characteristic curve and association with CAD. PTX3 may become a potentially powerful inflammatory biomarkers for the $C A D[30,31]$. It may provide a possibility for early screening of CAD patients independently of all classical risk pathways[32]. PTX3, a novel therapeutic and diagnostic target may provide an actionable opportunity for the precision cardiovascular medicine[33].

\section{Abbreviations}

MFGE8 Milk fat globulin E8

FC-AMs Free cholesterol-induced apoptotic macrophages

PTX3 Pentraxin 3

CR1 Complement receptor 1 
LDL Low-density Lipoprotein

$\operatorname{tg} 2$ trans-glutaminase 2

CAD Coronary artery disease

DCs Dendritic cells

GAS6 Growth arrest-specific protein 6

ACAT Acyl-CoA: cholesterol O - acyltransferase

PBS Phosphate-buffered saline

FACS Fluorescence-activated cell sorter

\section{Declarations}

\section{Ethical Approval and Consent to participate}

Yes

\section{Consent for publication}

All authors have seen the manuscript and approved to submit to your journal.

\section{Availability of supporting data}

No

\section{Funding}

This work was supported by the grants from the National Natural Science Foundation of P. R. China (No.81701376).

\section{Authors' contributions}

Data curation: Tang-meng Guo.

Software: Tang-meng Guo.

Supervision: Li Ke, Bei Cheng.

Writing - original draft: Tang-meng Guo.

All authors read and approved the final manuscript. 
Acknowledgements

We sincerely acknowledge Director Yong Zhou for writing assistance.

\section{Authors' information}

Prof. Bei Cheng, MD PhD

E-mail addresses: chengbei2014@126.com

Telephone/fax numbers: 086-027-85351552

Addresses: a Department of Geriatrics, Union Hospital, Tongji Medical College, Huazhong University of Science and Technology, Wuhan 430022, China; b Institute of Geriatrics, Union Hospital, Tongji Medical College, Huazhong University of Science and Technology, Wuhan 430022, China

Tang-Meng Guo, MD PhD

E-mail addresses: guotangmeng@126.com

Telephone/fax numbers: 086-027-85351545

Addresses: a Department of Geriatrics, Union Hospital, Tongji Medical College, Huazhong University of Science and Technology, Wuhan 430022, China; b Institute of Geriatrics, Union Hospital, Tongji Medical College, Huazhong University of Science and Technology, Wuhan 430022, China

Li Ke, , MD PhD

E-mail addresses: 334647954@qq.com

Telephone/fax numbers: 086-027-85351552

Addresses: a Department of Geriatrics, Union Hospital, Tongji Medical College, Huazhong University of Science and Technology, Wuhan 430022, China; b Institute of Geriatrics, Union Hospital, Tongji Medical College, Huazhong University of Science and Technology, Wuhan 430022, China

\section{Declarations}

The authors declare no conflicts of interest.

\section{References}

1. Norata GD, Marchesi P, Pulakazhi Venu VK, Pasqualini F, Anselmo A, Moalli F, et al. Deficiency of the long pentraxin ptx3 promotes vascular inflammation and atherosclerosis. Circulation. 2009; 
2. Rolph MS, Zimmer S, Bottazzi B, Garlanda C, Mantovani A, Hansson GK. Production of the long pentraxin PTX3 in advanced atherosclerotic plaques. Arterioscler Thromb Vasc Biol. United States; 2002;22:e10-4.

3. Kravitz MS, Pitashny M, Shoenfeld Y. Protective molecules - C-reactive protein (CRP), serum amyloid $P(S A P)$, pentraxin3 (PTX3), mannose-binding lectin (MBL), and apolipoprotein A1 (Apo A1), and their autoantibodies: Prevalence and clinical significance in autoimmunity. J. Clin. Immunol. 2005.

4. Scimeca M, Salustri A, Bonanno E, Nardozi D, Rao C, Piccirilli E, et al. Impairment of PTX3 expression in osteoblasts: a key element for osteoporosis. Cell Death Dis. England; 2017;8:e3125.

5. Savchenko AS, Imamura M, Ohashi R, Jiang S, Kawasaki T, Hasegawa G, et al. Expression of pentraxin 3 (PTX3) in human atherosclerotic lesions. J Pathol. 2008;215:48-55.

6. Shimizu T, Suzuki S, Sato A, Nakamura Y, Ikeda K, Saitoh S, et al. Cardio-protective effects of pentraxin 3 produced from bone marrow-derived cells against ischemia/reperfusion injury. J Mol Cell Cardiol. England; 2015;89:306-13.

7. Csukás D, Urbanics R, Wéber G, Rosivall L, Szebeni J. Pulmonary intravascular macrophages: Prime suspects as cellular mediators of porcine CARPA. Eur J Nanomedicine. 2015;7:27-36.

8. Guo T, Ke L, Qi B, Wan J, Ge J, Bai L, et al. PTX3 Is located at the membrane of late apoptotic macrophages and mediates the phagocytosis of macrophages. J Clin Immunol. 2012;32:330-9.

9. Haskard DO, Boyle JJ, Mason JC. The role of complement in atherosclerosis. Curr Opin Lipidol. 2008;19:478-82.

10. Pilely K, Bakke SS, Palarasah Y, Skjoedt MO, Bartels ED, Espevik T, et al. Alpha-cyclodextrin inhibits cholesterol crystal-induced complement-mediated inflammation: A potential new compound for treatment of atherosclerosis. Atherosclerosis [Internet]. Elsevier B.V.; 2019;283:35-42. Available from: https://doi.org/10.1016/j.atherosclerosis.2019.01.034

11. Cao W, Bobryshev Y V., Lord RSA, Oakley REI, Lee SH, Lu J. Dendritic cells in the arterial wall express C1q: Potential significance in atherogenesis. Cardiovasc Res. 2003;60:175-86.

12. Pulanco MC, Cosman J, Ho M-M, Huynh J, Fing K, Turcu J, et al. Complement Protein C1q Enhances Macrophage Foam Cell Survival and Efferocytosis. J Immunol. 2017;198:472-80.

13. Ji S-R, Wu Y, Potempa LA, Liang Y-H, Zhao J. Effect of modified C-reactive protein on complement activation: a possible complement regulatory role of modified or monomeric C-reactive protein in atherosclerotic lesions. Arterioscler Thromb Vasc Biol. United States; 2006;26:935-41.

14. Helmy KY, Katschke KJJ, Gorgani NN, Kljavin NM, Elliott JM, Diehl L, et al. CRIg: a macrophage complement receptor required for phagocytosis of circulating pathogens. Cell. United States; 2006;124:915-27.

15. Lee M-Y, Kim W-J, Kang Y-J, Jung Y-M, Kang Y-M, Suk K, et al. Z39Ig is expressed on macrophages and may mediate inflammatory reactions in arthritis and atherosclerosis. J Leukoc Biol. United States; 2006;80:922-8.

16. Seimon TA, Wang Y, Han S, Senokuchi T, Schrijvers DM, Kuriakose G, et al. Macrophage deficiency of p38a MAPK promotes apoptosis and plaque necrosis in advanced atherosclerotic lesions in mice. J 
Clin Invest. 2009;119:886-98.

17. Van Vre EA, Ait-Oufella H, Tedgui A, Mallat Z. Apoptotic cell death and efferocytosis in atherosclerosis. Arterioscler Thromb Vasc Biol. United States; 2012;32:887-93.

18. Schrijvers DM, De Meyer GRY, Kockx MM, Herman AG, Martinet W. Phagocytosis of apoptotic cells by macrophages is impaired in atherosclerosis. Arterioscler Thromb Vasc Biol. United States; 2005;25:1256-61.

19. Thorp E, Tabas I. Mechanisms and consequences of efferocytosis in advanced atherosclerosis. J Leukoc Biol. United States; 2009;86:1089-95.

20. Kojima Y, Weissman IL, Leeper NJ. The Role of Efferocytosis in Atherosclerosis. Circulation. United States; 2017;135:476-89.

21. Szka ZNP, Met KN, Csermely P Ter, Hudecz F, Mezo G, St GF. Defensins purified from human granulocytes bind $\mathrm{C} 1 \mathrm{q}$ and activate the classical complement pathway like the transmenbrane glycoprotein gq41 of HIV-1. Mol Immunol. 1997;34:809-16.

22. Taylor PR, Carugati A, Fadok VA, Cook HT, Andrews M, Carroll MC, et al. A Hierarchical Role for Classical Pathway Complement Proteins in the Clearance of Apoptotic Cells in Vivo. J Exp Med. 2000;192:359-66.

23. Bhatia VK, Yun S, Leung V, Grimsditch DC, Benson GM, Botto MB, et al. Complement C1q reduces early atherosclerosis in low-density lipoprotein receptor-deficient mice. Am J Pathol [Internet]. American Society for Investigative Pathology; 2007;170:416-26. Available from: http://dx.doi.org/10.2353/ajpath.2007.060406

24. Asperti C, Astro V, Pettinato E, Paris S, Bachi A, de Curtis I. Biochemical and functional characterization of the interaction between liprin-a1 and GIT1: implications for the regulation of cell motility. PLoS One [Internet]. 2011/06/13. Public Library of Science; 2011;6:e20757-e20757. Available from: https://www.ncbi.nlm.nih.gov/pubmed/21695141

25. Ajona D, Pajares MJ, Corrales L, Montuenga LM, Pio R. Activation of the classical complement pathway in lung cancer: A novel biomarker for diagnosis and prognosis. Immunobiology. 2012;217:1135.

26. Segerling M, Hahn H, Opferkuch W. Phagocytosis of C3b-coated sheep red blood cells by guinea-pig macrophages in the absence of antibody. Immunobiology. Netherlands; 1982;162:39-45.

27. Bohnsack JF, Oshea JJ, Takahashi T, Brown EJ. Fibronectin-enhanced phagocytosis of an alternative pathway activator by human culture-derived macrophages is mediated by the $\mathrm{C} 4 \mathrm{~b} / \mathrm{C} 3 \mathrm{~b}$ complement receptor (CR1). J Immunol. 1985;135:2680-6.

28. Kojima Y, Volkmer J-P, McKenna K, Civelek M, Lusis AJ, Miller CL, et al. CD47-blocking antibodies restore phagocytosis and prevent atherosclerosis. Nature. England; 2016;536:86-90.

29. Guo T-M, Huang L-L, Liu K, Ke L, Luo Z-J, Li Y-Q, et al. Pentraxin 3 (PTX3) promoter methylation associated with PTX3 plasma levels and neutrophil to lymphocyte ratio in coronary artery disease. J Geriatr Cardiol. 2016;13. 
30. Guo T-M, Ke L, Zhang X-E, Li L, Xiong L-J, Cheng B. Reply: Pentraxin-3 and coronary artery disease. Exp Gerontol. 2018;103.

31. Guo T, Huang L, Liu C, Shan S, Li Q, Ke L, et al. The clinical value of inflammatory biomarkers in coronary artery disease: PTX3 as a new inflammatory marker. Exp Gerontol [Internet]. 2017;97:64-7. Available from: http://linkinghub.elsevier.com/retrieve/pii/S0531556517300529

32. Shanker J, Kakkar V V. Contribution of classical and emerging risk factors to coronary artery disease in Asian Indians. Int J Cardiol. Netherlands; 2016;214:97-106.

33. Antman EM, Loscalzo J. Precision medicine in cardiology. Nat Rev Cardiol. 2016;13:591-602.

\section{Figures}
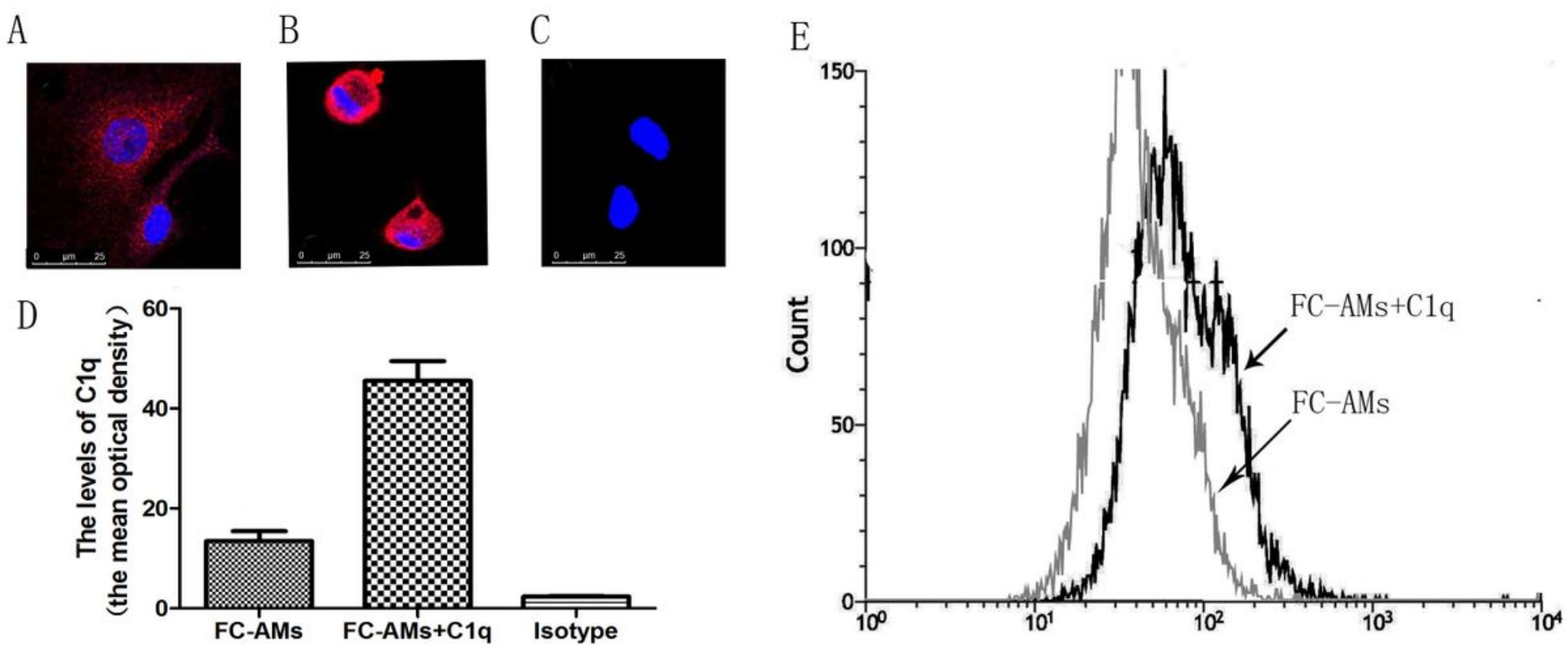

Figure 1

Fig. 1. Binding of C1q to FC-AMs. FC-AMs were generated by incubating C57BL6/J mice macrophages with $100 \mu \mathrm{g} / \mathrm{ml}$ of acetyl-LDL and $10 \mu \mathrm{g} / \mathrm{ml}$ of the compound 58035. To harvest these FC-AMs, a nonenzymatic cell dissociation solution was used to treat FC-AMs at $37^{\circ} \mathrm{C}$ for $5-10 \mathrm{~min}$. Next the FC-AMs were incubated with $\mathrm{C} 1 \mathrm{q}$ for $30 \mathrm{~min}$ at $4^{\circ} \mathrm{C}$. The control group was treated in the absence of $\mathrm{C} 1 \mathrm{q}$. Then the FC-AMs were washing with RPMI 1640 supplementing with $10 \%$ fetal bovine serum. After incubating with goat serum, the FC-AMs were incubated with anti-C1q mAbs for 30 min at $4^{\circ} \mathrm{C}$. The membrane surface protein $\mathrm{C} 1 \mathrm{q}$ were recognized with anti-C1q $\mathrm{mAbs}$ and the secondary antibody (goat anti-mouseCy3-labeled IgG) (red-orange). C1q was detected on the membrane surface of FC-AMs (A). Strong binding of $\mathrm{C} 1 \mathrm{q}$ was detected when FC-AMs were incubated with $\mathrm{C} 1 \mathrm{q}(\mathrm{B})$. As a negative control isotype (isotypematched control mAb of $\mathrm{C} 1 \mathrm{q}$ ), the fluorescence intensity of cells was lowest (C). The levels of $\mathrm{C} 1 \mathrm{q}$ were reflected by the mean optical density, which was analyzed using Image-Pro Plus software. The statistical results were shown in D. Scale bar represents $25 \mu \mathrm{m}$ in all images. Bars indicate the mean $\pm \mathrm{SE}$. As expected, C1q levels were higher in pre-incubation with C1q group in FACS (E). 


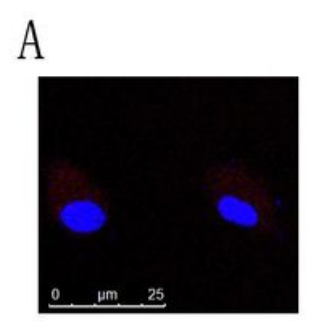

B
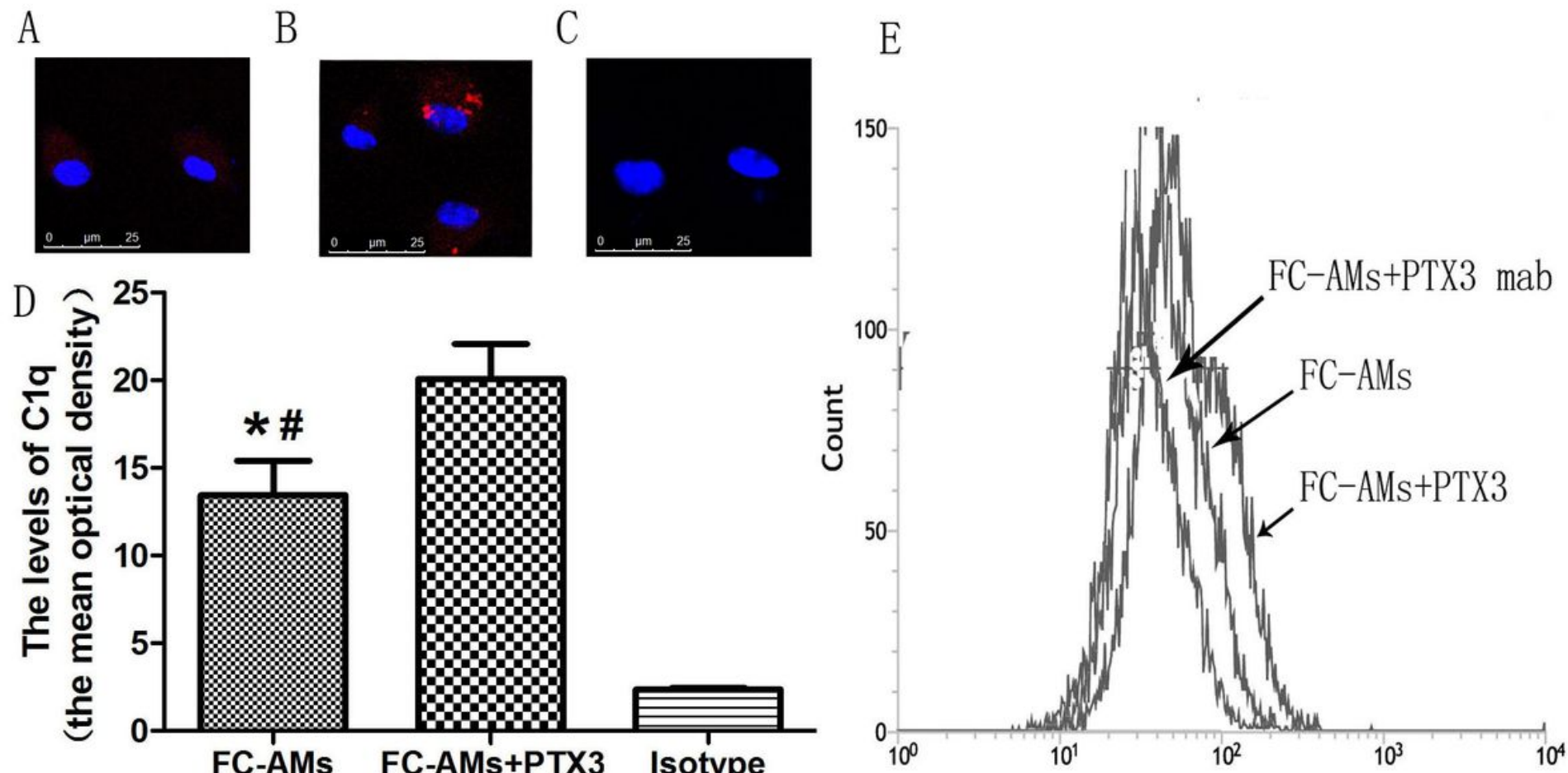

Figure 2

PTX3 enhances C1q binding on apoptotic cells. FC-AMs were incubated with PTX3 or anti-PTX3 mAbs for 30 min at $4^{\circ} \mathrm{C}$. Then the FC-AMs were washing with RPMI 1640 supplementing with $10 \%$ fetal bovine serum. After incubating with goat serum, the FC-AMs were incubated with anti-C1q mAbs for $30 \mathrm{~min}$ at $4^{\circ} \mathrm{C}$. The membrane surface protein $\mathrm{C} 1 \mathrm{q}$ were recognized with anti-C1q mAbs and the secondary antibody (goat anti-mouse-Cy3-labeled IgG) (red-orange). The mean optical density of $\mathrm{C} 1 \mathrm{q}$ (red-orange) in FC-AMs groups was $13.45 \pm 2.54(\mathrm{~A})$, lower than that in FC-AMs+PTX3 groups $(20.06 \pm 3.00)(\mathrm{B})$, and more than Isotype $(2.36 \pm 1.03)(C)$. The statistical results were shown in $D$. It showed more percentages of $\mathrm{C} 1 \mathrm{q}$ positive cells pre-incubated with PTX3. Scale bar represents $25 \mu \mathrm{m}$ in all images. As expected, C1q levels were highest in pre-incubation with PTX3 group in FACS (E). The effects of PTX3 on C1q binding on apoptotic cells were reversed with anti-PTX3 mAbs. Compared to the FC-AMs+PTX3 groups (the percentage of $\mathrm{C} 1 \mathrm{q}$ positive cells was $0.90 \% \pm 0.29 \%$ ), the percentage of $\mathrm{C} 1 \mathrm{q}$ positive cells (pre-incubation with anti-PTX3 mAbs groups) was $0.75 \% \pm 0.44 \%$ (mean $\pm \mathrm{SE}, \mathrm{n}=3, \mathrm{P}<0.01$ ). 

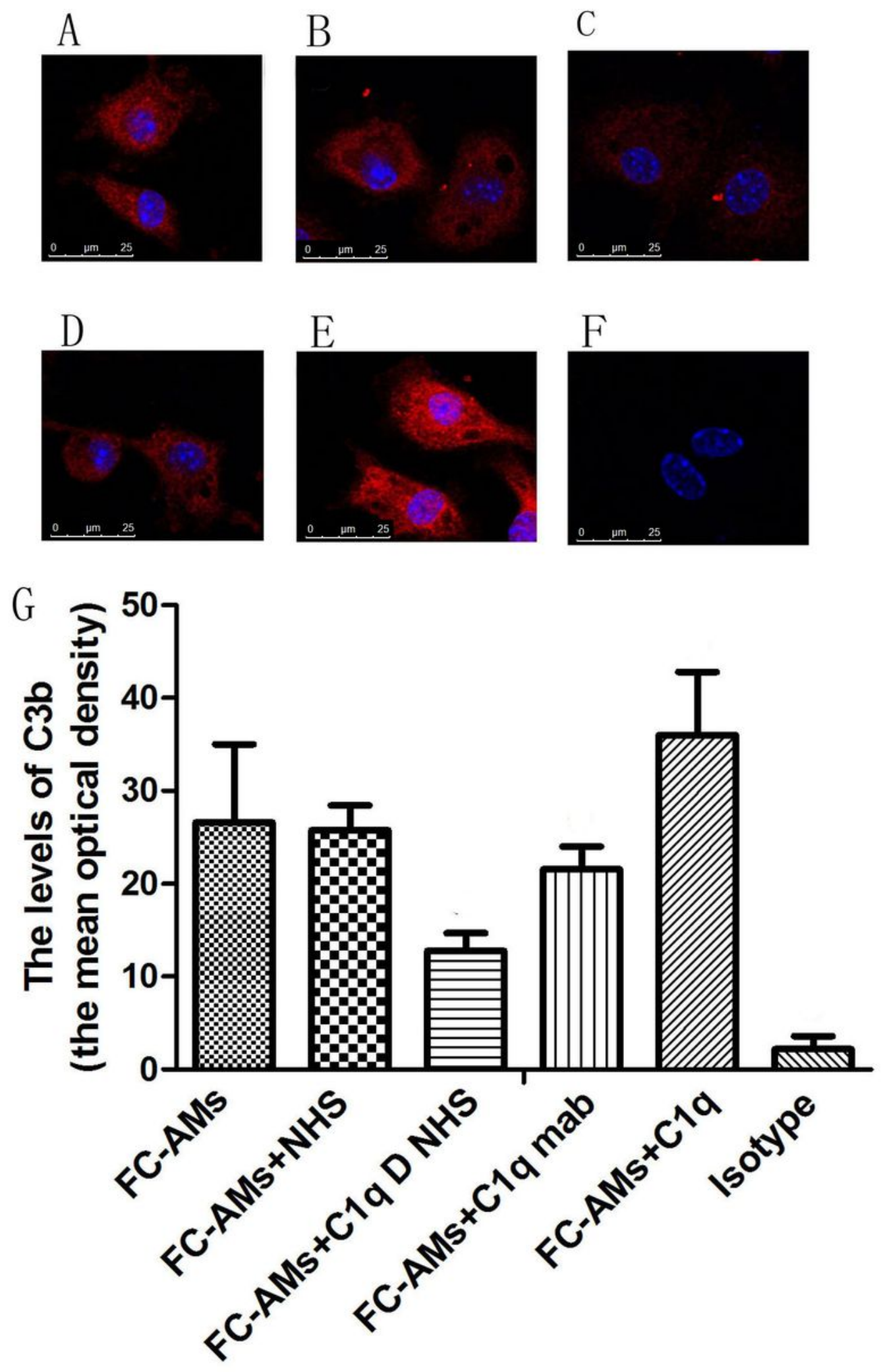

Figure 3

C1q-mediated activating of classic complement pathway. FC-AMs were treated with NHS, C1q D NHS, anti-C1q mAbs and C1q. MAbs to C3b was used to detect C3b deposition on the FC-AMs. FC-AMs alone (A). FC-AMs with NHS (B), FC-AMs with C1q D NHS (C), FC-AMs with anti-C1q mAbs (D), FC-AMs with C1q $(E)$, Isotype as a negative control $(F)$. The C3b deposition was inhibited significantly in the absence of 
C1q (C, D). It was reversed by incubating with C1q (E). Scale bar represents $25 \mu \mathrm{m}$ in all images. The statistical results presented as mean \pm SD were shown in $\mathrm{G}$.
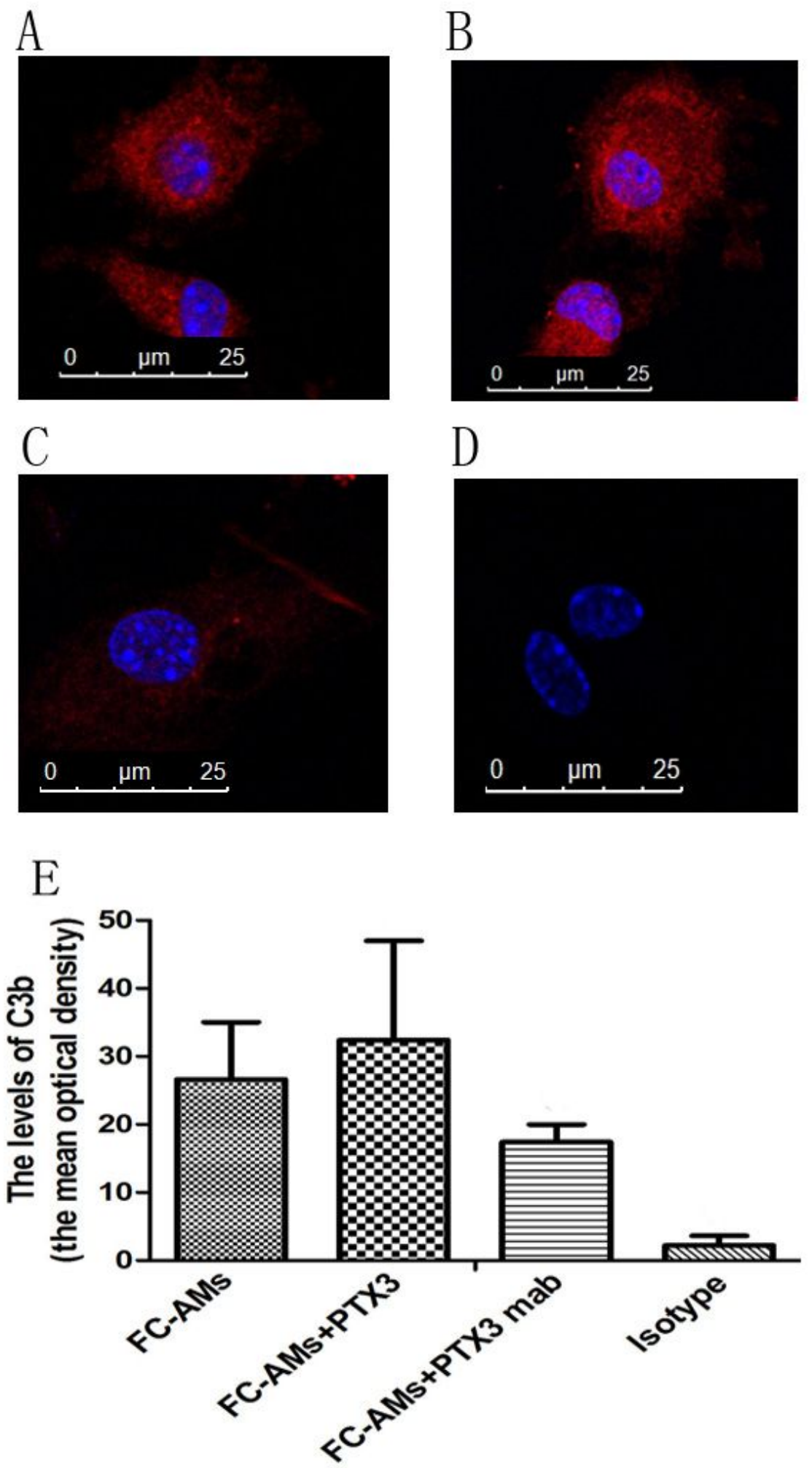

Figure 4

PTX3 enhances C1q-mediated C3b deposition. FC-AMs were incubated with PTX3 or anti-PTX3 mAbs for 30 min at $4^{\circ} \mathrm{C}$. Then the FC-AMs were washing with RPMI 1640 supplementing with $10 \%$ fetal bovine serum. MAb to C3b was used to detect C3b deposition on the FC-AMs (A). C3b deposition was increased 
when incubating FC-AMs with PTX3(B). Pre-incubation with anti-PTX3 mAbs resulted in significant C3b decline on the FC-AMs(C). Isotype as a negative control (D). Scale bar represents $25 \mu \mathrm{m}$ in all images. The statistical results presented as mean \pm SD were shown in $\mathrm{E}$.

A
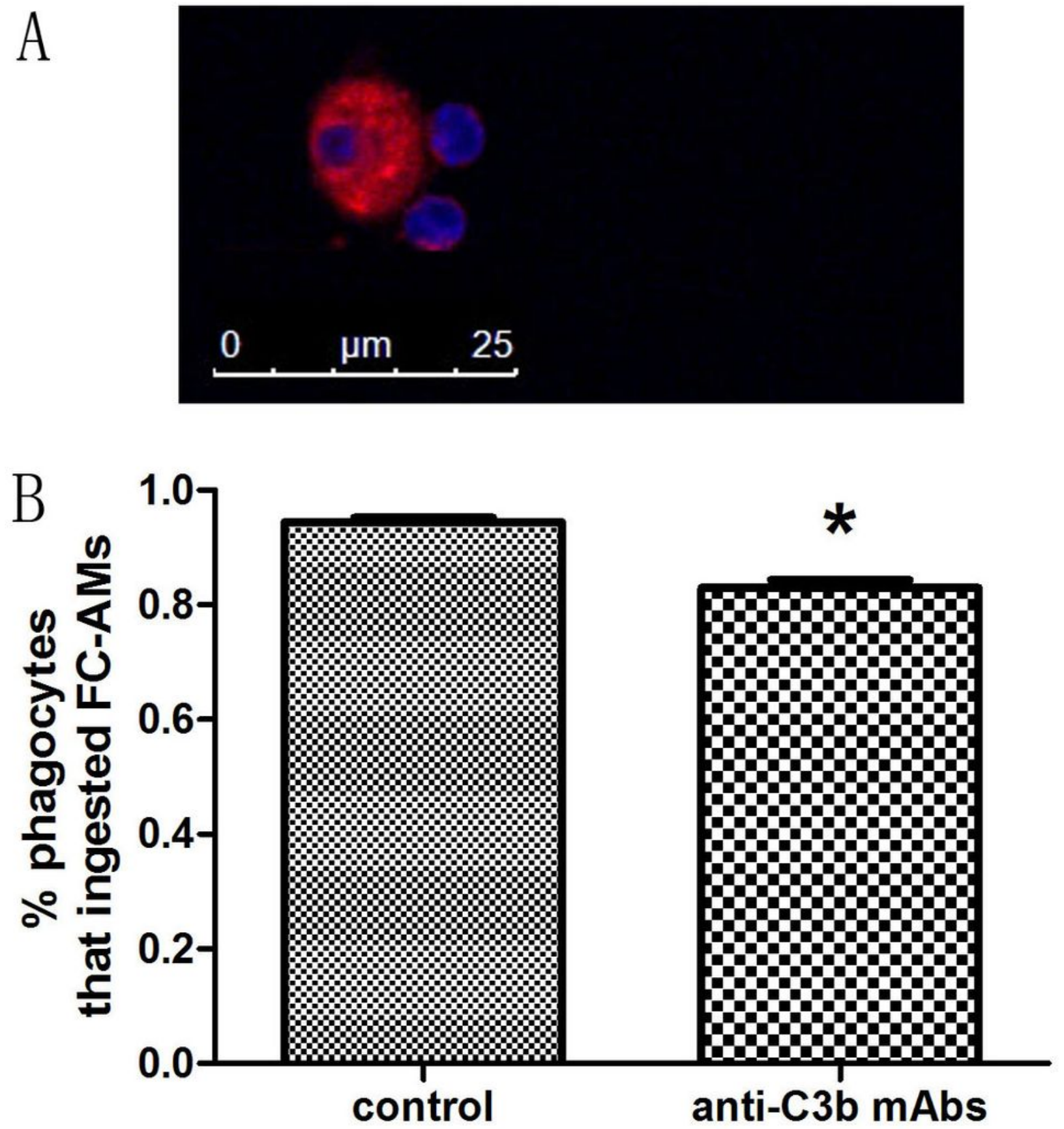

Figure 5

Inhibitory effect of anti-C3b mAbs on the phagocytosis of FC-AMs by macrophages. A, FC-AMs were prelabeled with the membrane probe DiL(red-orange). FC-AMs were added to a monolayer of phagocytes for 
30 min. Non-ingested cells were removed by thorough washing, and then they were stained with DAPI(blue) and fixed, viewed by confocal fluorescence microscopy. Scale bar represents $25 \mu \mathrm{m}$ in all images. B, The effect of anti-C3b mAbs on the phagocytosis of FC-AMs by peritoneal macrophages. Phagocytosis was scored by counting the number of ingested FC-AMs. The percentage of phagocytes that ingested FC-AMs was quantified. It showed phagocytosis was inhibited by anti-C3b mAbs. Asterisks refer to the statistical significance versus control groups: ${ }^{*} \mathrm{P}<0.05$.

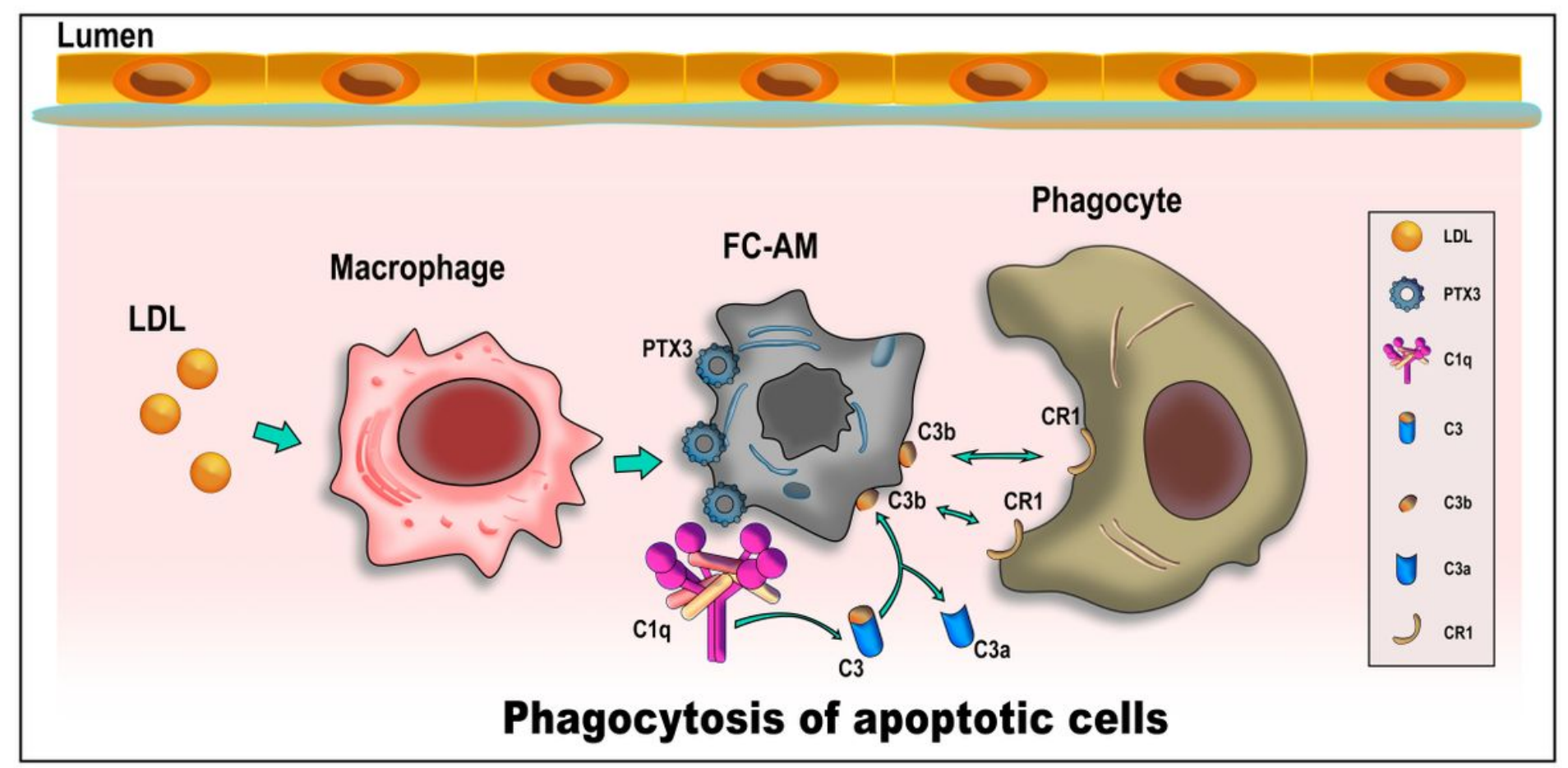

Figure 6

The series processes. 